\title{
Projection Technique for Vortex-Free Image Registration
}

\author{
Patrick Scheibe $^{1}$, Ulf-Dietrich Braumann ${ }^{1,2}$, Jens-Peer Kuska ${ }^{2}$ \\ ${ }^{1}$ Translational Center for Regenerative Medicine (TRM) \\ ${ }^{2}$ Interdisciplinary Center for Bioinformatics \\ University Leipzig, Germany \\ Email: mai99dnn@studserv.uni-leipzig.de
}

\begin{abstract}
One important application of image processing in medicine is to register tissue samples onto another. Registering these highly textured images with non-parametric methods sometimes leads to solutions which are known to be suboptimal. We show a projection technique for unwanted vortices in the displacement field. A new strategy is presented which does not change the registration method itself but continuously correct the solution during the registration process.
\end{abstract}

\section{Introduction}

Many variants of non-parametric image registration methods are known [1]. The basic goal is to find a transformation $\boldsymbol{u}(\boldsymbol{x})$ so that the transformed template image $T(\boldsymbol{x}-\boldsymbol{u}(\boldsymbol{x}))$ is similar to the reference image $R(\boldsymbol{x})$. This similarity which indirectly is expressed in the difference of the two images is not the only condition the searched transformation has to fulfill. Beside this, a smoothing term is part of the registration criterion which is physically motivated, whereas its task is to ensure the smoothness of the transformation. This method, which was introduced in [2], applied to high textured tissue samples still may lead to vortex-affected solutions.

One may ask how to know that these solutions are bad. The explanation is: In the way of getting the image data from the medical device, some kind of image deformations are very unlikely. Especially vortices which are often part of the solutions with high textured images are demonstrably false and should not be part of the displacement field $\boldsymbol{u}(\boldsymbol{x})$. These are caused by the solution procedure when images with many similar structures are used. Tissue consisting of many cells is one example for highly textured data.

A first workaround for the vortex problem was presented by Kuska and Braumann [3]. Their method includes the suppression of vortices as weighted term in the variational problem.

In this paper we present a new method to get rid of these vortices. It is based on the idea of projecting them continuously out during the iterative steps of finding the solution $\boldsymbol{u}(\boldsymbol{x})$. 


\section{Methods}

Vortex-free image registration extends a curvature-based method that was introduced in [4] and later used in [1]. Braumann and Kuska discussed in [2] the influences of the boundary conditions and gave a fast implementation for the method. The registration follows a variational approach to find the transformation $\boldsymbol{u}(\boldsymbol{x})$ that fulfills

$$
\min _{\boldsymbol{u}}(\mathcal{D}(\boldsymbol{u})+\alpha \mathcal{S}(\boldsymbol{u}))
$$

with

$$
\begin{aligned}
\mathcal{D}(\boldsymbol{u}) & \left.=\frac{1}{2} \int_{\Omega}(T(\boldsymbol{x}-\boldsymbol{u}(\boldsymbol{x})))-R(\boldsymbol{x})\right)^{2} d \boldsymbol{x} \\
\mathcal{S}(\boldsymbol{u}) & =\frac{1}{2} \int_{\Omega}(\Delta \boldsymbol{u})^{\mathrm{T}} \cdot(\Delta \boldsymbol{u}) d \boldsymbol{x}
\end{aligned}
$$

With the rules of the calculus of variations it follows a highly non-linear partial differential equation which was solved by extending it toward a time dependent function

$$
\frac{\partial \boldsymbol{u}}{\partial t}(\boldsymbol{x}, t)=-\alpha \Delta^{2} \boldsymbol{u}(\boldsymbol{x}, t)+\boldsymbol{f}(\boldsymbol{x}, \boldsymbol{u}(\boldsymbol{x}, t))
$$

with

$$
\boldsymbol{f}(\boldsymbol{x}, \boldsymbol{u}(\boldsymbol{x}, t))=(R(\boldsymbol{x})-T(\boldsymbol{x}-\boldsymbol{u}(\boldsymbol{x}, t))) \cdot \nabla T(\boldsymbol{x}-\boldsymbol{u}(\boldsymbol{x}, t))
$$

This function $\boldsymbol{u}(\boldsymbol{x}, t)$ converges with $\lim _{t \rightarrow \infty} \boldsymbol{u}(\boldsymbol{x}, t)=\boldsymbol{u}(\boldsymbol{x})$. Equation 3 is solved with an iterative algorithm. The only place where vortices can arise is the term $\boldsymbol{f}(\boldsymbol{x}, \boldsymbol{u}(\boldsymbol{x}, t))$.

What our new method does is to project all vortices out of this term before the next time-step is calculated. This is possible using the theorem of Helmholtz which states a vector field can be decomposed in its irrotational and its solenoidal component. With this it is possible to calculate the solenoidal part of $\boldsymbol{f}(\boldsymbol{x}, \boldsymbol{u}(\boldsymbol{x}, t))$ and subtract it. This vortex-free version is then used in the calculation of the next time-step.

The extraction of the solenoidal part $\nabla \times \boldsymbol{p}$ is again a variational problem given by the functional

$$
\mathcal{W}[\boldsymbol{p}]=\frac{1}{2} \int_{\Omega}|\nabla \times \boldsymbol{p}-\boldsymbol{f}|^{2} d \boldsymbol{x}
$$

The partial differential equation that is defined by this functional is a Poisson equation for the third element of the vector potential $\boldsymbol{p}=(0,0, p)^{\mathrm{T}}$

$$
\frac{\partial^{2}}{\partial x^{2}} p(\boldsymbol{x})+\frac{\partial^{2}}{\partial y^{2}} p(\boldsymbol{x})=\frac{\partial}{\partial y} f_{1}(\boldsymbol{x})-\frac{\partial}{\partial x} f_{2}(\boldsymbol{x})
$$

Since we have digital images, we have to solve a discrete version of this equation. To avoid the calculation of a linear system of equations in $p$ we use the Fourier transform with appropriate boundary conditions. Therefore only one equation per point must be solved. 
Fig. 1. The original image and the distorted image with the applied frog- eye transformation. The advantage of this transformation is the fact that the inverse is known and one can identify solution errors easily
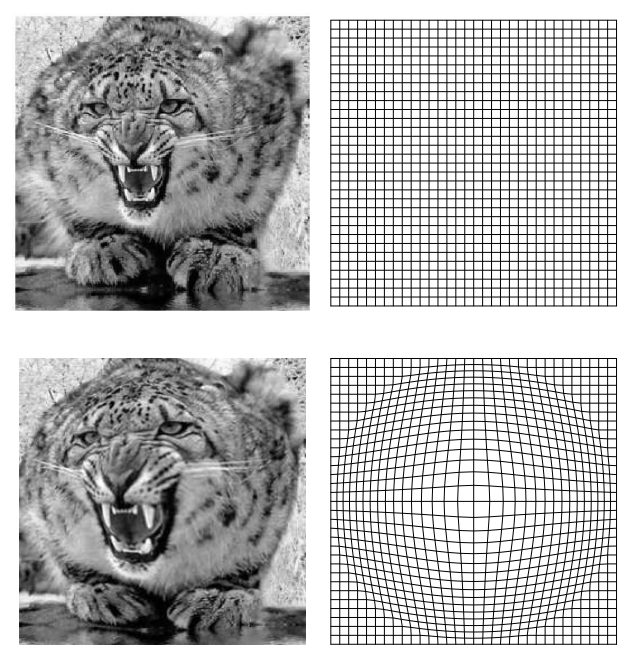

\section{Results}

As input for the first illustrative example an image is given that was distorted with a vortex-free transformation. Thus, the solution of the registration problem should not contain vortices either. Since the registration method may run into unwanted vortices when the image is highly textured, we have distorted a picture of a leopard whose coat is obviously textured enough (see fig. 1). In figure 2 the pictures in the upper row depicts the registration without vortex extraction. Notice that the high textured region of the leopards head causes the method to distort this area. That can be clearly seen looking at the line integral convolution [5] in the middle picture column. The difference between the transformation found by the method and the real inverse is given by the error plots in the right column of figure 2 .

In contrast to that with our new strategy this critical region of the head of the leopard is handled very well. Only the error plot unveils where the method is not exactly. The human eye is not able to find differences between the original and the registered image.

In figure 3 we present the application of our new method on a realistic problem. The images show two HE-stained histological slices of the uterine cervix which are registered onto another. As one can clearly see, the right line integral convolution shows a much smoother displacement field. This was calculated using the new vortex-free registration. 

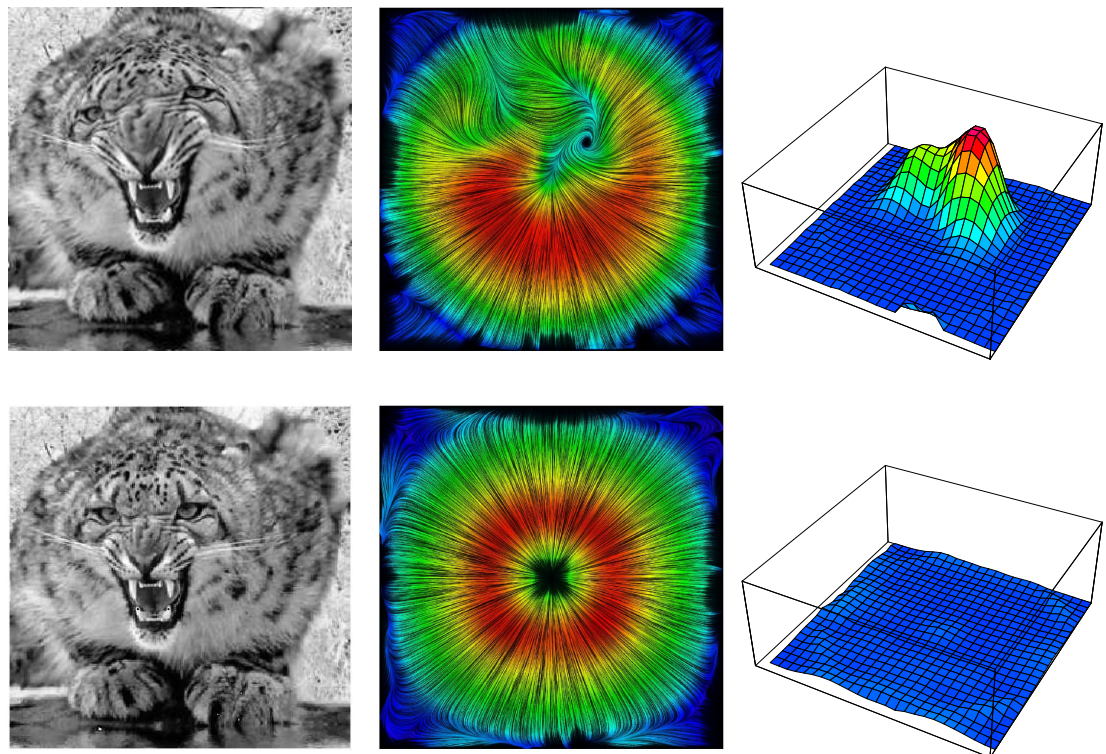

Fig. 2. Comparison of the method without (upper row) and with (lower row) vortex extraction: results after registration (left), line integral convolutions of the displacement fields (middle), error plots for the obtained displacement vector fields (right)
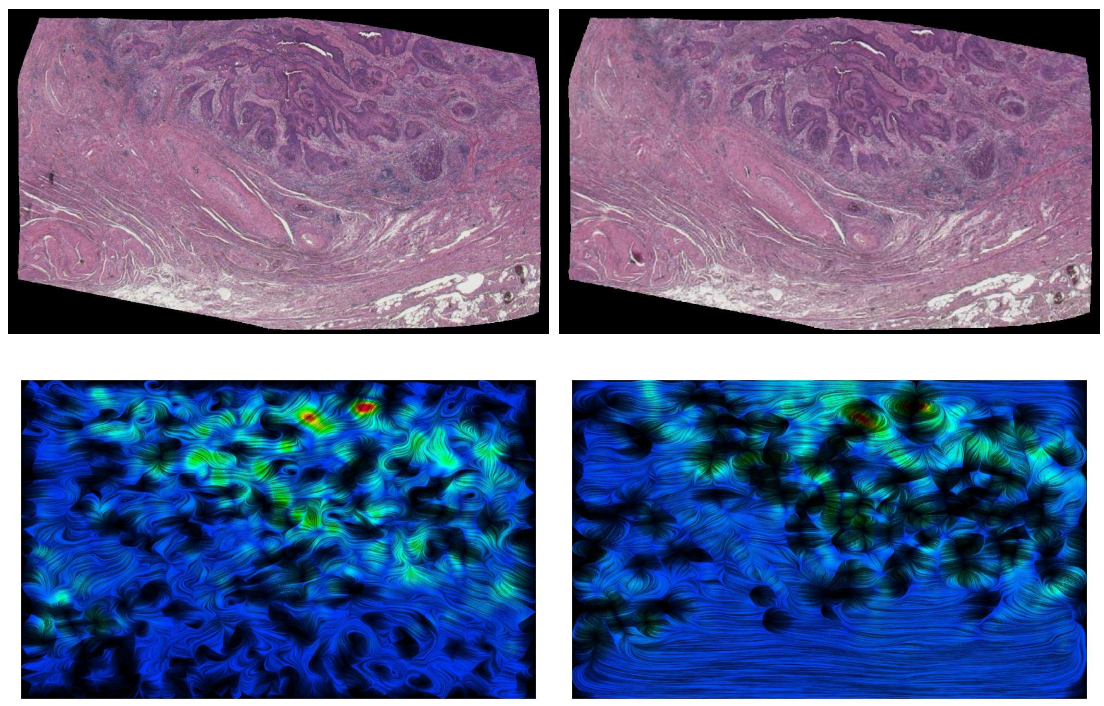

Fig. 3. Two HE-stained histological slices consecutively cut from a specimen of a squamous cell carcinoma of the uterine cervix, which are to be registered (top) and displacement field (bottom): without vortex extraction (left), using our new algorithm (right). As in fig. 2, blue denotes weak, green medium, and red strong displacements 


\section{Discussion}

We have presented a projection technique for image registration that allows to force the displacement field to be vortex-free. This is appropriable in cases where it is known that the solution does not contain vortices. The method presented in [3] has several drawbacks compared to our new approach:

- The vortex suppression term in the functional can lead to situations where its influence is on a level disabling the terms for minimal distance and smoothness. Our strategy leaves the functional as is, so this situation is not possible.

- Even when the term for vortex suppression is rated high it is still possible that the calculation runs into a solution where vortices appear in the displacement field. Since we project them out of the partial solution and let the method find a good transformation in the space of vortex-free functions this can not happen.

- The projection that extracts all vortices does not need a weighting parameter like the vortex suppression term. Therefore our method has one less parameter to adjust.

Since the Helmholtz decomposition requires sufficiently smooth and fast decaying vector fields, the border of the images can be a bit problematic. For vortices near the border the decomposition does not work well but by adding extra space around the image it is likely that we can suppress this behavior.

Finally we can say that the presented algorithm surpasses our expectations and yields in all test cases better results than the underlying curvature-based registration.

\section{References}

1. Modersitzki J. Numerical Methods for Image Registration. Oxford University Press, USA; 2004.

2. Braumann UD, Kuska JP. Influence of the boundary conditions on the result of nonlinear image registration. In: Proceedings of the IEEE International Conference on Image Processing. IEEE Signal Processing Society; 2005. I-1129-I-1132.

3. Braumann UD, Kuska JP. A new equation for nonlinear image registration with control over the vortex structure in the displacement field. In: Proceedings of the IEEE International Conference on Image Processing. IEEE Signal Processing Society; 2006. 329-332.

4. Yali A. A nonlinear variational problem for image matching. SIAM Journal on Scientific Computing 1994;15(1):207-224.

5. Cabral B, Leedom LC. Imaging vector fields using line integral Convolution. In: Proceedings SIGGRAPH '93; 1993. 263-272.

6. Braumann UD, Kuska JP, Einenkel J, Horn LC, Löffler M, Höckel M. Threedimensional reconstruction and quantification of cervical carcinoma invasion fronts from histological serial sections. IEEE Trans Med Imag 2005;24(10):1286-1307. http://dx.doi.org/10.1109/TMI.2005.855437. 